\title{
¡Quo Vadis Joven! Relatos del abuso de drogas en jóvenes de Guayaquil, Ecuador
}

\section{Quo Vadis Young! Stories of drug abuse in young people in Guayaquil, Ecuador}

\section{Quo Vadis Young! Histórias de abuso de drogas em jovens em Guayaquil, Equador}

Franco Coffré, $\mathbf{J}^{1}$., Oviedo Rodríguez, $\mathbf{R}^{2}$., Donoso Triviño, $\mathrm{A}^{3}$., Piedra Chavez, $\mathrm{K}^{4}$., \& Cujilan Alvarado, M.C. ${ }^{5}$

${ }^{1}$ Magíster en Gerencia en Servicios de la salud. Universidad de Guayaquil. Correo electrónico: joicy.francoc@ug.edu.ec

${ }^{2}$ Magíster en Educación Superior. Universidad de Guayaquil. Correo electrónico: ruth.oviedor@ug.edu.ec

${ }^{3}$ Magíster en Gerencia en salud para el desarrollo local. Universidad de Guayaquil. Correo electrónico: Alicia.donosot@ug.edu.ec

${ }^{4}$ Doctora en Ciencias Pedagógicas. Universidad de Guayaquil. Correo electrónico: piedrack@ug.edu.ec

${ }^{5}$ Magister en Gerencia en salud para el desarrollo local. Universidad de Guayaquil. Correo electrónico: maira.cujilana@ug.edu.ec

Cómo citar este artículo en edición digital: Franco-Coffré, J., Oviedo-Rodríguez, R., DonosoTriviño, A., Piedra-Chávez K. y Cujilán-Alvarado M. (2020). ¡Quo Vadis Joven! Relatos del abuso de drogas en jóvenes de Guayaquil, Ecuador. Cultura de los Cuidados (Edición digital), 24 (56)

Recuperado de http://dx.doi.org/10.14198/cuid.2020.56.03

Correspondencia: La Romareda Etapa 4 Maz 55 villa 13, Guayaquil, Ecuador.

Correo electrónico de contacto: joicy.francoc@ug.edu.ec

Recibido:12/09/2019

Aceptado:20/12/2019

\section{ABSTRACT}

Introduction: The abuse of drugs is an important public health problem not only in Ecuador, but also worldwide. Objective: To interpret the perceptions of the drugdependent person about the effects that the drug produces in their personal, family, social life, and their vision for the future. Methodology: Qualitative research with phenomenological interpretative analysis performed by semi-structured interviews in non-probabilistic sampling snowball type to 17 subjects with drug dependence in the period July-August 2018 in the city of Guayaquil. Results: The key informants were between 16 and 28 years of age, with low social stratum, from marginal urban 
areas, mostly incomplete secondary education, and with a history of first contact

with the drug in early adolescence and in educational institution. Five categories were obtained: Family context, and the community environment as causal factors of consumption; social discrimination as punishment for the addicted person; guilt, loneliness and low self-esteem; the train of addiction to drugs destroys in its path: health and life; and family support and a Supreme Being as alternatives to get out of addiction. Conclusions: The causes of drug use were among others: the escape from dysfunctional family problems and adolescent vulnerability in their search for identity and belonging to a group. On the other hand, they reported feeling discriminated against and punished by society, and they foresee that a future of death, prison, sickness and indigence awaiting them.

Key words: Personality disorders, family life, perception, drug abuse, social problems.

\section{RESUMEN}

Introducción: El uso indebido de drogas es un importante problema de salud pública no solo en el Ecuador, sino también a nivel mundial. Objetivo: Interpretar las percepciones de la persona drogodependiente sobre los efectos que produce la droga en su vida personal, familiar, social, y su visión para el futuro. Metodología: Investigación cualitativa con análisis interpretativo fenomenológico realizado mediante entrevistas semiestructuradas en muestreo no probabilístico tipo bola de nieve, a 17 sujetos con drogodependencia, en el periodo julio-agosto del 2018, en la ciudad de Guayaquil. Resultados: Los informantes clave, tenían entre 16 a 28 años de edad, estrato social bajo, procedentes de zonas urbano-marginales, en su mayoría de instrucción secundaria incompleta, y con antecedentes de primer contacto con la droga en la adolescencia temprana y en la institución educativa. Se obtuvieron cinco categorías: contexto familiar y el entorno comunitario como factores causales del consumo; discriminación social como castigo a la persona adicta; culpa, soledad y autoestima baja; el tren de la adicción a las drogas destruye a su paso: salud y la vida; y

el apoyo familiar y de un Ser Supremo como alternativas para salir de la adicción. Conclusiones: Las causas del consumo de drogas fueron, entre otras: el escape de los problemas de su familia disfuncional y la vulnerabilidad adolescente en su búsqueda de identidad y pertenencia a un grupo. Por otra parte, relataron que se sienten discriminados y castigados por la sociedad, y vislumbran que les espera un futuro de muerte, cárcel, enfermedad e indigencia.

Palabras clave: Trastornos de la personalidad, vida familiar, percepción, toxicomanía, problema social.

\section{RESUMO}

Introdução: $\mathrm{O}$ abuso de drogas é um grande problema de saúde pública, não apenas no Equador, mas em todo o mundo. Alvo: Interpretar as percepções da pessoa dependente de drogas sobre os efeitos produzidos pela droga em sua vida pessoal, familiar, social e sua visão para o futuro. Metodologia: Pesquisa qualitativa com análise interpretativa fenomenológica realizada por meio de entrevistas semiestruturadas em amostragem sem probabilística de bolas de neve, para 17 sujeitos com dependência de drogas, no período de julho a agosto de 2018, na cidade de Guayaquil. Resultados: Os informantes chave tinham entre 16 e 28 anos de idade, baixos estratos sociais, de áreas urbanas marginais, principalmente ensino médio incompleto, e com uma história de primeiro em contato com drogas no início da adolescência e na instituição de ensino. Cinco categorias foram obtidas: contexto familiar e o ambiente da comunidade como fatores causais do consumo; discriminação social como punição para a pessoa viciada; culpa, solidão e baixa auto-estima; o trem do tóxico dependência destrói o seu caminho: saúde e vida; e apoio familiar e um Ser Supremo como alternativas para sair do vício. Conclusões: as causas do uso de drogas foram, entre outros: a fuga de problemas familiares disfuncionais e a vulnerabilidade do adolescente em sua busca 
por identidade e pertencimento a um grupo. Por outro lado, relataram que se sentem discriminados e punidos pela sociedade, e prevêem que um futuro de morte, prisão, doença e indigência os espera.

Palavras chave: Transtornos de personalidade, vida familiar, percepção, toxicodependência, problema social.

\section{INTRODUCCIÓN}

Uno de los más grandes problemas sociales y de salud a nivel mundial es el consumo de sustancias estupefacientes. Al menos el 5\% de la población adulta en el mundo ha consumido drogas por lo menos una vez en el año 2015, por otro lado, el daño provocado se representa en 28 millones de años de vida sana perdidos como resultado del consumo de drogas (UNODC, 2017). En el Ecuador se identifica que la edad promedio de inicio del consumo de drogas está entre 14 y 15 años de edad; y que las drogas de fácil adquisición son la marihuana, heroína o $\mathrm{H}$. La droga $\mathrm{H}$ tiene entre sus componentes hasta un 80\% de heroína, y el resto que es el 20\% lo componen cafeína, diltiazem, cocaína, pasta base y otras sustancias más, se identificó adicionalmente que “en el 2015 se suscitaron 8.693 fallecimientos relacionados al consumo de drogas, lo que representa el 13,42\% del total de fallecimientos a nivel nacional” (SETED, 2017, pp. 109-110).

Los adolescentes son la población más susceptible en el inicio del consumo de drogas. Según el Instituto Nacional de abuso de Drogas de los Estados Unidos, el 20\% de los estudiantes de 12do. grado dijeron que podían obtener heroína fácilmente si lo deseaban (Johnson, O’Malley, Miech, Bachman y Schulenberg, 2016). Casi 1 de cada 4 adolescentes en el Ecuador (24\%), alguna vez se ha embriagado y el $29 \%$ ha visto usar drogas a algún estudiante del colegio. Además, al 15\% de adolescentes les sería fácil conseguir marihuana; al 8\% comprar heroína; al 6\%, cocaína; al 4\%, éxtasis; y al 3\%, pasta base. Llama la atención que el $46 \%$ considera que en su centro educativo circulan drogas: el 24\% dice que ha visto a algún estudiante que las vende o las pasa, y el 48\% afirma que alrededor de su colegio o escuela venden estupefacientes (Observatorio social del Ecuador, 2016). Al tener contacto con la droga en edades tempranas, los adolescentes se ven envueltos en un riesgo alto de convertirse en adictos. El cerebro en desarrollo no puede formarse adecuadamente bajo la influencia de las drogas (Munro, 2015).

Entre las causales del consumo de drogas se encuentra de forma preponderante las carencias, esto se refiere a las necesidades afectivas y un perfil parental que refleje autoridad en la familia. Por otro lado, la crisis del futuro que percibe el joven es otro factor de riesgo importante, ya que, al no tener un proyecto de vida establecido, hipoteca su futuro al consumo de drogas. Adicionalmente se encuentra la personalidad adictiva (Chinneck et al., 2018), que pertenece a una estructura y tipo familiar 
Revista científica de la Asociación de Historia y Antropología de los Cuidados (Universidad de Alicante)

disfuncional (Vargas et al., 2015), las creencias asociadas al consumo (Vargas y Londoño, 2016), la curiosidad (Heydari et al, 2015), los pares (Lessa et al., 2014), la narcotización de la comunidad en la que viven (Potrikeeva, Suprun, Ispulova, Oleinik y Slepkhina, 2017) el alivio al estrés, tristeza y depresión (Dejman et al., 2015) y la facilidad de adquisición de la droga en la institución educativa a la que asisten (Simões, Gaspar, Batista-Foguet y SimonsMorton, 2014; Neto, Fraga y Ramos, 2012). Por otro lado, entre las consecuencias clínicas del uso abusivo de drogas, se encuentran la depresión respiratoria (opiáceos) (Cohen y Weinstein, 2018), quemaduras en vías respiratorias, neumotórax, neumomediastino, síndrome coronario e infarto agudo de miocardio (cocaína y crack) (Devlin y Henry, 2008); consecuencias a nivel de salud mental como episodios de psicosis (Basu y Parakh, 2017) y también las consecuencias sociales como son la exposición a conductas sexuales de riesgo (Khajedaluee, Dadgarmoghaddam, Erfanian, Alipourtabrizi y KhademRezaiyan, 2015; Bosma-Bleeker y Blaauw, 2018) y violencia física (Abdalla et al., 2017) y sexual (Carneiro de Camargo, Romancini, Schneider y Ferraz, 2017) entre otras. La drogodependencia y los trastornos que provoca se han considerado como un problema de salud pública, y esta problemática ha sido puesta a consideración ya que se encuentra en la meta 3.5 del Objetivo de Desarrollo Sostenible 3 en la cual se insta a los gobiernos para que fortalezcan la prevención y el tratamiento del abuso de sustancias adictivas (OMS, 2017).

La persona drogodependiente es etiquetada por la sociedad como una persona peligrosa (Corrigan, Kuwabara y O’Shayghnessy, 2009) adicta, indeseable y de la cual hay que tener distancia (Luo et al., 2014). Pocos consumidores de drogas tienen acceso a recibir tratamiento de rehabilitación y, cuando acceden, existen factores que podrían llevarlos a abandonar los servicios ambultarorios de drogodependecncias, como el ser mujer, ser joven y ser consumidor de cocaína (Calvo et al., 2017) por otro lado, el hecho de vivir con su pareja e hijos, y poseer un nivel medio de autoestima son factores predictores de mayor adherencia (Andrés, 2017). No obstante, cuando el tratamiento fracasa o es abandonado existe la opción de acceder a un programa de Reducción de daños, el cual es aplicado en ciertos países del mundo y que consiste básicamente en la prescripción de Metadona y Buprenorfina como tratamiento a la adicción de los opiáceos, distribución de jeringuillas estériles e instalación de salas de consumo supervisados (Bosque-Prous y Brugal, 2016).

El objetivo general de esta investigación, es poder interpretar las percepciones que tiene la persona drogodependiente sobre los efectos que 
produce la droga en su vida, tanto en el contexto familiar, social, personal como su visión para el futuro.

\section{METODOLOGÍA}

La naturaleza de este estudio es de enfoque cualitativo, de tipo fenomenológico y analítico. Se aplicó en una muestra de 17 pacientes consumidores de drogas que cumplían con los criterios de inclusión: 1) Historia de consumo de drogas mayor a 1 año, 2) hombres y mujeres de edades entre 15 a 30 años, 3) no haber sido ingresado nunca en un centro de rehabilitación. Como criterios de exclusión: 1) Historia de consumo de drogas menor a 1 año, 2) hombres y mujeres de edades menores a 15 años ni mayores a 30 años, 3) nunca haber estado ingresado en un centro de rehabilitación.

Para la elección de los sujetos de investigación se aplicó un muestreo intencional de bola de nieve o en cadena, en pacientes que compartían experiencias importantes similares y que podrían referir otros casos ricos en información (Polgar y Thomas, 2014). Los primeros sujetos captados fueron identificados como pacientes de Centros de salud del Ministerio de salud pública del Ecuador, y estos una vez captados y habiendo aceptado participar voluntariamente en el estudio, referían otras personas consumidoras para así poder aplicarles también la entrevista. El total de sujetos de investigación obtenido en esta muestra fue de 17 sujetos hasta la saturación de los datos.

Para la realización de la entrevista fueron utilizados -en ciertas ocasiones- los domicilios de los sujetos de investigación a puerta cerrada para evitar distracciones o sonidos que pudiesen distorsionar la grabación de audio, en otros casos se lo realizó en parques aledaños o en el mismo centro de salud. Hubo necesidad de cerciorarse previamente que los participantes no se encontrasen con efectos de la droga y poder tener respuestas certeras. Se iniciaba mediante un saludo cordial y con la presentación del entrevistador, y mediante el lenguaje corporal se trató de fomentar un clima desinhibido y de confianza, así como aclarar dudas sobre el objetivo de la investigación y que puedan conocer que no se vulneraría su intimidad ni revelaría su identidad por la delicadeza del tema.

Las preguntas de la entrevista semiestructuradas tenían 5 enfoques a cubrir que son: a) Datos sociodemográficos, b) Historia pasada del consumo de droga, c) Motivación para el consumo de droga, d) Vida de la persona que consume droga, e) Ventajas y desventajas percibidas por el consumo de drogas-Perspectivas de la vida a futuro. El detalle de las preguntas se encuentra en la tabla 1. Las entrevistas continuaron hasta la saturación de datos. El análisis de la información utilizó enfoque inductivo, codificación abierta y codificación selectiva mediante metodología de teoría fundamentada, construcción de las 
Revista científica de la Asociación de Historia y Antropología de los Cuidados (Universidad de Alicante)

categorías en base a la codificación y el contexto de los fenómenos que vivenciaron los sujetos de investigación, y posteriormente la aproximación teórica.

Se transcribieron textualmente el audio de las entrevistas (datos brutos), para posteriormente realizar el análisis de contenido inductivo: se leyó en varias ocasiones las respuestas para tener una idea completa y se seleccionaron las frases más llamativas y paradigmáticas. Posterior a ello se colocaron los códigos para luego formular las subcategorías y categorías mediante la interpretación del contexto de cada afirmación extraída.

Se incluyeron criterios de rigor metodológico:

credibilidad, confirmabilidad, significado en contexto, y transferibilidad. Como parte de la ética de la investigación, se solicitó la revisión y aprobación por parte del Comité de Ética de la Facultad de Ciencias Médicas de la Universidad de Guayaquil previo a la aplicación de las entrevistas, así como la explicación previa a los sujetos de estudio sobre el objetivo de la investigación, y que sus identidades serían salvaguardadas y mantenidas en anonimato. Para ello se hizo firmar el formato de consentimiento informado de la Universidad de Guayaquil.

\section{RESULTADOS Y DISCUSIÓN}

Los informantes clave, tenían de 16 a 28 años de edad, estrato bajo, procedentes de zonas urbano marginales de la ciudad de Guayaquil. En su mayoría de instrucción secundaria incompleta, y con antecedentes de primer contacto con la droga en la adolescencia temprana y en la institución educativa. De los 17 informantes, 16 eran hombres y una sola mujer, todos desempleados y en ocasiones en condición de indigencia y abandono.

\section{Contexto familiar, $y$ el entorno comunitario como factores causales del consumo}

La familia es la unidad sustantiva de la sociedad, es donde el niño crece y forma su carácter de acuerdo a la educación, trato, y ambiente percibido dentro del hogar. Según las unidades de análisis producidas por las entrevistas realizadas, llama la atención que las relaciones familiares disfuncionales fueron las circunstancias que inclinaron al consumo de sustancias ilícitas a tempranas edades. Entre ellas un informante declaró como causa de consumo de drogas: "Porque ese man de mi padrastro me pegaba me obligaba a lavar carro, le pegaba a mi mamá y ella se dejaba y mis panas me decían que así olvidaba todo" Aquarius, 28A.

En ocasiones la persona drogodependiente mencionaba inferencias sobre sentimiento de culpa y reconocimiento de su equivocación, así como dotaba de culpa de su disfunción familiar a la sociedad, porque de acuerdo a su criterio, esta facilitaba o no 
Cultura de los Cuidados

impedía adecuadamente el tráfico de sustancias ilegales.

"Fue desde la parte mía el consumo de droga fue porque tenía una mente equivocada de pensar me dejaba llevar de la sociedad, me dejaba llevar por lo que veía, por cosas, que por problemas familiares, también con mis padres, con mi madre, cosas que me agobiaban, opté por entrar al consumo activo de drogas, era borrego de las demás personas” Lepus, 18A.

En los Estados Unidos se ha reportado que los eventos potencialmente traumáticos en la infancia están asociados con el riesgo de consumo de drogas ilícitas entre los adolescentes de este país ${ }^{\mathrm{i}}$. Este hallazgo representa un comportamiento modificable y que se consideraría como foco de intervención para la prevención del uso y abuso de sustancias ilícitas.

También mencionaron circunstancias como las pérdidas familiares: "me sentía mal mentalmente después de la pérdida de mi abuela, desde ahí comencé a consumir drogas" Geminis, 19A. "Falta de hombría, porque busqué lo más fácil, dizque por fugarme, me enteré de una verdad muy dura para mí, aparte, perdí a la mujer que quería en ese tiempo y perdí a la figura paterna, fue todo junto" Aquila, 22A. La disgregación familiar es el principal problema dentro de los usuarios de sustancias ilícitas, aparición de conflictos, perdida de vínculos y lazos afectivos destruidos con las personas significativas, son muy comunes cuando existe esta problemática (Ferreira et al., 2017).
Según la Teoría de Campo de Lewin (citado en Bahia, Furtado y Trassi, s. f.), menciona que la conducta está en función de las relaciones que se establecen entre la persona y su ambiente. Este ambiente es el resultado de la configuración de factores interdependientes que conforman el espacio vital constituido por espacios positivos $\mathrm{y}$ negativos, así como fuerzas que nos atraen y nos repelen. Esta teoría permite la comprensión de los cambios conductuales que enfrenta la juventud y la influencia que tienen los pares en el consumo de drogas.

\section{Discriminación social como castigo a la persona adicta}

El consumo de drogas, es uno de los problemas que generan mayor rechazo social, debido a que se percibe a la persona que consume como un ente delictivo, desadaptado y de cierta peligrosidad (Losantos, Berckmans, Pieters, Dómic y Loots, 2015). Sin embargo, este desprecio colectivo, provoca en el drogodependiente sentimientos de soledad, culpa $y$, en ocasiones, resentimiento hacia la sociedad, lo que puede desencadenar en actos violentos hacia quienes lo rodean; o, por el contrario, en aislamiento (no empleo, no residencia, no lazos familiares) (Duarte, Antunes, Moraes, Olschowsky y Barbosa de Pinho, 2016). Estos estigmas y prejuicios, lejos de lograr en la persona adicta una concientización hacia su situación, fortalece aún más la conducta de consumo, dificultando su reintegro a la sociedad, a la vez que se forma un círculo vicioso del cual 
Revista científica de la Asociación de Historia y Antropología de los Cuidados (Universidad de Alicante)

es muy difícil escapar. Un dato interesante es aquel que relaciona a la discriminación hacia el individuo con el índice de abandono del tratamiento de rehabilitación para salir del consumo de drogas, el cual es proporcional, es decir, a mayor discriminación, mayor deserción. (Brener, Hippel, Hippel, Resnick y Treloar. 2010).

“Mi mamá me trata mal, me dice mi papá que soy lo peor, todo el mundo me mira mal y me siento mal por eso” Leo, 18A.

En estas reacciones parentales se está reconociendo la imposibilidad de poder ejercer algún tipo de control sobre el adicto, es sabido que los psicólogos muchas veces recomiendan a los familiares que es mejor hacerlos a un lado para que el resto de la familia pueda seguir una vida de tranquilidad. ¿y el papel de los padres? ¿acaso se pierde cuando existen problemas de consumo de droga?, como mecanismo de defensa, el padre se despersonaliza y deja a un lado ese rol, adquiere una postura de darse por vencido con la esperanza de que el joven pueda salir de aquel mundo y así no tener que afrontar directamente la situación. El egoísmo, la insensibilidad, rechazo y los estereotipos sociales sobre las personas que consumen drogas es evidente (Rengel, 2005). Las redes sociales, la televisión y la prensa sensacionalista, estigmatizan a la persona que consume drogas como alguien peligroso, sin principios ni valores y de poca valía, dos informantes manifestaron:

"Ya no te ven igual, te ven diferente y tienen miedo de que hagas otra cosa y cometas otro error, y así” Aquarius, 28A.

"La gente me ve como cucaracha (suspira), como si fuera bicho raro, como que si no valiera la pena” Andromeda, 16A.

“Me siento mal, porque la gente me juzga por lo que consumo y habla cosas de mí. Ya mis amigos no existen, me fallaron, solo los que consumen conmigo” Hydra, 19A.

“En el momento que comencé a consumir droga mi vida cambió, comencé a hacer cosas, cosas antisociales, comencé a robar, comencé a llevarme las cosas de mi hogar, comencé a faltarle el respeto a mi madre, perdí el respeto a mí mismo” Lepus, 18A.

La construcción social del otro afecta notablemente a la persona drogodependiente, en su autoestima, rol de ciudadano, hijo y de ser humano. Touzé (1995) menciona que hay tres tipos de representaciones sociales: Distorsión, Suplementación y la Reducción o rebaja. Esta última corresponde a la supresión de atributos perteneciente al objeto, lo cual se traduce a la represión de las normas sociales. La persona drogodependiente es considerada como un ente que posee nulo autocontrol y como consecuencia representa una amenaza para la sociedad por su identidad deformada.

Culpa, soledad y autoestima baja como pensamientos que martillan la cabeza del consumidor

Entre los factores personales que influyen significativamente en el consumo 
de drogas se encuentra la baja autoestima

(Alavi, 2011), que incide en los pensamientos negativos que muchas veces presentan los adolescentes, unido a los desajustes emocionales propios de su fase etaria, entre ellos el deseo de independencia, periodos de hostilidad, y autosuficiencia.

"Lo pierdes todo, nadie te respeta todos piensan que vales trozo" Aquarius, 28A.

“Me siento bien volando, pero así cuando regreso me siento como que mal, a veces porque creo que estoy haciendo algo incorrecto, pero igual no me importa porque a fin de cuentas hay que vivir, y así empecé el camino no puedo dejarlo atrás, yo he visto panas que se van y que dicen que van a dejar de consumir y lo logran, y otros que no, entonces yo no quiero intentar tampoco sentirme mal" Centaurus, 24A.

“Al principio me sentía bacán, me sentía chévere, sentía monstruoso, pero ya después, ya esta nota como que ya cansa, me botaron de la casa mi familia, no me quieren ni ver, entonces ya pues, yo sé que en algún momento tengo que cambiar, pero todavía no es tiempo" Eridanus, 20A.

Pese al reconocimiento del problema que tienen y se autoperciben como adictos, no desean apartarse de ese círculo infernal como el descrito por Dante, se dice que los drogadictos no aparecen o nacen de la nada, no se generan espontáneamente, son el producto de privaciones, limitaciones y carencias que se dan en los hogares, familia, el barrio, así como de la violencia social que observan desde temprana edad.
El tren de la adicción a las drogas destruye a su paso: la salud y la vida

A poco tiempo del consumo de las drogas ilegales puede ya notarse los efectos adversos. Se incluyen la taquicardia, depresión respiratoria en el caso de los opioides, entre otras. Ya el efecto adverso a largo plazo podría conllevar al consumidor a padecer de nefropatías, cirrosis, y algunas patologías cardiacas. Con respecto a ello se mencionan las siguientes narrativas sobre cómo el consumo de drogas afecta su salud: "Pues afecta mi salud, mi cuerpo, me ahogo al respirar, yo sé que tarde o temprano acabará con mi vida, pero aun así es peor dejarla” Andromeda, 16A.

"Me afecta en todo, desde que te digo que no estoy con mi familia y todo eso, eso me afecta bastante pero ya pues la nota no se la puede dejar" Eridanus, 20A.

"Me destruí, me puse flaco, me llené de ojeras, muchas veces tenía lagunas mentales, olvidaba las cosas, comencé a perder el sentido de la vista, el de degustar porque todo comenzó a hacerse igual" Lepus, 18A.

La adicción es la fuerza motriz más tenaz que el conocimiento del daño autoinflingido al cuerpo. La adicción es un fenómeno que implica múltiples orígenes: genético, psicológico, ambiental y educacional (Rojo-Mota, 2016). Esta conducta adicta, repetitiva, escapa de la supervisión cerebral y altera el funcionamiento de diversas estructuras 
Revista científica de la Asociación de Historia y Antropología de los Cuidados (Universidad de Alicante)

cerebrales, a manera de ejemplo es como que o la cárcel” Geminis, 19A.

si el cerebro sufriera un apagón que favorece a la pérdida del control de la persona adicta. Donas (2001), médico salubrista Esto explica como los informantes clave del estudio pese a reconocer los daños físicos que ha causado el consumo de drogas, no pueden abandonarlo.

En otro aspecto, las personas drogodependientes, vislumbran un futuro muy oscuro caracterizado por indigencia, cárcel, enfermedad y muerte. Para Travelbee, las capacidades humanas universales que distinguen a un ser humano, son "el hecho de tener un propósito o sentido en la vida, $\mathrm{y}$ un significado en el sufrimiento” (citada en Galvis, 2015, p. 1115). De ahí nacen las preguntas ¿qué sentido en la vida tiene la persona drogodependiente? y ¿qué perspectiva de futuro tiene la persona consumidora? Las narrativas de los informantes fueron muy específicas y desgarradoras, totalmente impregnadas de sentimientos de desesperanza, prediciendo su final de los días en la calle, asesinados, enfermos y solos.

"Que me puedan matar como a perro" Aries, 30A.

"Sé que algún día terminaré bajo el puente sin ropa, comida, ni rumbo fijo, pero es que drogarme es la solución a mis problemas” Australis, 23A.

“Lo que me espera es la huesuda” Draco, 28A.

“El único futuro que me espera es la muerte pediatra, plantea que existen dos tipos de factores protectores: de amplio espectro y los específicos a ciertas conductas. Los factores protectores de amplio espectro son aquellos que otorgan más probabilidad de que no se haga realidad la conducta de riesgo, entre ellos se nombran a la familia funcional, la autoestima alta, las relaciones interpersonales positivas, la permanencia en el sistema educativo, $\mathrm{y}$ donde nos detendremos en el análisis: el proyecto de vida del adolescente fuertemente internalizado.

En un estudio realizado en Chile donde pretendieron abordar los factores de riesgo y protección de los adolescentes, mediante técnica de recolección de datos de grupos de discusión, evidenciaron en sus resultados que los adolescentes reconocieron que la familia, los amigos más cercanos y tener un proyecto de vida (carrera profesional, matrimonio, familia y trabajo) serían los factores protectores de mayor peso en sus vidas (Páramo, 2011).

\section{Apoyo Familiar y de un Ser Supremo como alternativas para escapar de la adicción}

A la familia se la considera como la principal red de apoyo para la retención del paciente drogodependiente en un programa 
de rehabilitación.

"Que me apoyaran, que no me ataquen y me digan que haga con mi vida lo que quiera, si pudiera cambiaría para poder estar con mi hijo, esa sería una motivación, cambiar por mi hijo y con la bendición de Dios poder salir del consumo" Sagitarius, 16A.

Al haber la droga deteriorado y en ocasiones anulado el rol de padre, la persona adicta puede tomar como incentivo para su recuperación, la figura de sus hijos. Sin embargo, recuperar su rol parental de forma efectiva y la funcionalidad de su familia depende de factores internos y externos, tales como el apoyo familiar (que muchas veces ya ha perdido la confianza por los continuos fracasos de la persona drogodependiente y ante ello se niega a ayudar) y el factor económico, ya que por medio de este se podría solventar el servicio brindado por los centros de adicciones.

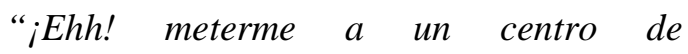
rehabilitación no creo, porque en los centros de rehabilitación le dan las drogas y los mandan a robar, en las noches los sueltan y no es bueno los centros de rehabilitación. Preferible para mí que me encierren en mi casa, que me encadenen para no salir en las noches; me pongan sueros y el parche que se pone para desintoxicar” Taurus, 19A.

“Que me lo proponga y que mi madre esté siempre conmigo, y que Papá Dios no me desampare, proponérmelo y seguir los puntos que sí se puede salir de las drogas y seguir” Aquila, 22A.
La rehabilitación de la persona adicta está directamente asociada al sistema familiar, a los suprasistemas de la sociedad, suprasistema político, las relaciones interpersonales, y también el suprasistema religioso. Aducen que un Ser Superior es el único que podría obrar el milagro que no han conseguido realizar por sus propios medios, $\mathrm{y}$ que esa fuerza poderosa y paranormal podría resolver todos los problemas adquiridos por el consumo de sustancias ilícitas.

En su gran mayoría los participantes manifestaron reconocer a un Ser Supremo y a la figura materna como protagonistas en su cambio verdadero. No obstante, dos de los participantes mencionaron que, pese a su reconocimiento del problema de la adicción, consideran sentirse a gusto y no tener deseos de abandonar el consumo. Entre las limitaciones que tuvo esta investigación, se consideró que algunas de las declaraciones de los participantes, fueron muy escuetas y cortantes a las preguntas de la entrevista. Pese a que el entrevistador quiso obtener más información, no había la fluidez de palabras ni deseos de colaboración más allá de lo indagado. Además, se deja para el análisis, si debiese sectorizarse o limitarse a un área geográfica destinada para la recolección de datos, y no haber tomado de varios sectores de la ciudad de Guayaquil, y deja la interrogante de que, si esta sectorización hubiese arrojado otro tipo de resultados. 
Revista científica de la Asociación de Historia y Antropología de los Cuidados (Universidad de Alicante)

CONSIDERACIONES FINALES

Los discursos comúnmente

enunciados como posibles causas de ingreso al mundo del consumo de drogas fueron: el escape de los problemas de su familia disfuncional y la vulnerabilidad adolescente en su búsqueda de identidad y pertenencia a un grupo. Como consecuencias del abuso de sustancias ilícitas, describieron el sentirse discriminados, rechazados, castigados por la sociedad, y con sentimientos de culpa y sensación de soledad. Lo que es peor, la perspectiva futura que tienen estos jóvenes al vivir en este mundo, es una vislumbre de muerte, cárcel, enfermedad e indigencia, pero dentro de la poca esperanza que verbalizan, otorgan a un Ser Supremo y a su familia la fuerza necesaria para poder rehabilitarse y ser nuevamente insertados en la sociedad.

\section{BIBLIOGRAFÍA}

Abdalla, R., Massaro, L., Queiroz de, M., Laranjeira, R., Caetano, R. y Madruga, C.(12 de noviembre, 2017). Association between drug use and urban violence: Data from the II Brazilian National Alcohol and Drugs Survey (BNADS). Addict Behavoir Reponse, 7, 8-13. Recuperado de https://www.ncbi.nlm.nih.gov/pubmed/295 $\underline{0250}$

Alavi, H. (verano/otoño, 2011). The Role of Self-esteem in Tendency towards Drugs, Theft and Prostitution [Resumen]. Addiction \& Health, 3(3-4), 119-124. Recuperado de https://www.ncbi.nlm.nih.gov/pubmed/249 $\underline{4126}$

Andrés de, S. (marzo, 2017). Factores asociados con la adherencia al tratamiento en pacientes con drogodependencias. Metas de Enfermería, 20(2), 18-24. Recuperado de http://www.enfermeria21.com/revistas/meta rticulo/81031/factores-asociados-con-laadherencia-al-tratamiento-en-pacientes-condrogodependencias/

Bahia, A., Furtado, O. y Trassi, M. (2001). Psicologias. Uma introdução ao estudo de Psicologia. Recuperado de https://petpedufba.files.wordpress.com/201 6/02/bock psicologiasumaintroduc3a7c3a3o-p.pdf

Basu, D. y Parakh, P. (6 de enero, 2017). Cannabis and Psychosis: Correlation, Causality and Consequences [Resumen]. En V. R. Preedy (Ed.), Handbook of Cannabis and Related Pathologies. Biology, Pharmacology, Diagnosis, and Treatment (pp. 413-421). doi: https://doi.org/10.1016/B978-0-12-800756$\underline{3.00048-X}$

Bosma-Bleeker, M. y Blaauw, E. (2018). Substance use disorders and sexual behavior; the effects of alcohol and drugs on patients' sexual thoughts, feelings and behavior. Addictive Behaviors, 87, 231-237. Recuperado de https://www.ncbi.nlm.nih.gov/pubmed/300 77915

Bosque-Prous, M. y Brugal, M. (2016). Intervenciones de reducción de daños en usuarios de drogas: situación actual y recomendaciones. Gaceta Sanitaria, 30(1), 99-105. doi:

https://doi.org/10.1016/j.gaceta.2016.04.02 $\underline{0}$

Brener, L., Hippel, W., Hippel, C, Resnick, I. y Treloar, C. (2010). Perceptios of discrminatory treatment by staff as predictor of drug treatment completion: Utility of a mixed methods approach. Drug and Alcohol Review, 29(5),491-497. doi: https://doi.org/10.1111/j.14653362.2010.00 173.x

Calvo, F., Carbonell, X., Valero, R., Costa, J., Turró, O., Giralt, C. y Ramírez, M. 
(2018). Abandono precoz y retención en servicios ambulatorios de drogodependencias: análisis transversal comparativo de factores que aumentan o disminuyen la adherencia. Atención Primaria, 50(8), 477-485. doi: https://doi.org/10.1016/j.aprim.2017.06.006

Carliner, H., Keyes, K., McLaughlin, K., Meyers, J., Dunn, E. y Martins, S. (2016). Childhood Trauma and Illicit Drug Use in Adolescence: A Population-Based National Comorbidity Survey ReplicationAdolescent Supplement Study. Journal of the American Academy of Child \& Adolescent Psychiatry, 55(8), 701-708. doi: https://doi.org/10.1016/j.jaac.2016.05.010

Carneiro de Camargo, J., Romancini, F., Schneider, L. y Ferraz, L. (2017) Consequences of drug use: perspective of the adolescent in rural areas. Revista de Pesquisa Cuidado é Fundamental Online, 9(4), 1028-1033. Recuperado de http://www.redalyc.org/articulo.oa?id=5057 $\underline{54110020}$

Chinneck, A., Thompson, K., Mahu, I., Davis-MacNevin, P., Dobson, K. y Steward, S. (2018). Personality and prescription drug use/misuse among first year undergraduates. Addict Behavoir, 87, 122-130. Recuperado de

https://www.ncbi.nlm.nih.gov/pubmed/300 05334

Cohen, K. y Weinstein, A. (2018). Synthetic and Non-Synthetic Cannabinoid Drugs and Their Adverse Effects-A Review from Public Health Prospective [Documento en línea]. Frontiers in Public Health, 6(162). Recuperado de https://www.frontiersin.org/articles/10.3389 /fpubh.2018.00162/full

Corrigan, P., Kuwabara, S. y O'Shaughnessy, J. (2009). The Public Stigma of Mental Illness and Drug Addiction. Findings from a Stratified Random Sample. Journal of Social Work, 9(2), 139-47. Recuperado de http://journals.sagepub.com/doi/abs/10.117 $\underline{7 / 1468017308101818}$
Dejman, M., Vameghi, M., Roshanfekr, P., Dejman, F., Rafiey, H., ... Johnson, R. (2015). Drug Use among Street Children in Tehran, Iran: A Qualitative Study. Frontiers in Public Health, 3(279). doi: https://doi.org/10.3389/fpubh.2015.00279

Devlin, R. y Henry, J. (2008). Clinical review: Major consequences of illicit drug consumption [Documento en línea]. Critical Care, 12(202). Recuperado de https://ccforum.biomedcentral.com/articles/ $\underline{10.1186 / \text { cc6166 }}$

Donas, S. (Comp.). (2001). Adolescencia y juventud en América Latina. Recuperado de http://www.binasss.sa.cr/adolescencia/Adol escenciayjuventud.pdf

Duarte, N., Antunes, B., Moraes, C., Olschowsky, A. y Barbosa de Pinho, L. (28 de marzo, 2016). Stigma and prejudice: the experience of crack users. RLAE. Revista Latino-Americana de Enfermagem, 24, 1-7. Recuperado de http://www.scielo.br/pdf/rlae/v24/es_01041169-rlae-0852-2680.pdf

Ferreira, V., Fernandes, M., Guimarães, A., Fernandes, J., Próspero, T. y Villar, M. (2017). Percepción de las mujeres en situación de dependencia química dentro de Mato Grosso, Brasil. Cultura de los Cuidados, 21(48), 33-42. Recuperado de https://rua.ua.es/dspace/bitstream/10045/69 270/1/CultCuid 48 04.pdf

Galvis, M. (2015). Teorías y modelos de Enfermería usados en la Enfermería Psiquiátrica. Revista Cuidarte, 6(2). 1108$1120 . \quad$ Recuperado de https://revistacuidarte.udes.edu.co/index.ph p/cuidarte/article/view/172/454

Heydari, S., Izedi, S., Sarikhani, Y., Kalani, N., Akbary, A., Miri, A., ... Akbari, M. (20 de junio, 2015). The Prevalence of Substance use and Associated Risk Factors Among University Students in the City of Jahrom, Southern Iran [Resumen]. International Journal of High Risk Behaviors and Addiction, 4(2), 22381. Recuperado de 
Revista científica de la Asociación de Historia y Antropología de los Cuidados (Universidad de Alicante)

https://www.ncbi.nlm.nih.gov/pubmed/260 97836

Johnston, L., O’Malley, P., Miech, R., Bachman, J. y Schulenberg, J. (2017). 2016 Overview Key Findings on Adolescent Drug Use. Recuperado de http://www.monitoringthefuture.org/pubs/m onographs/mtf-overview2016.pdf

Khajedaluee, M., Dadgarmoghaddam, M., Erfanian, M., Alipourtabrizi, A. y KhademRezaiyan, M. (2015). Women, Drug Dependency and Consequences: A Study from a Developing Country. Journal of Addiction, 2015, 2-6. doi: http://dx.doi.org/10.1155/2015/831954

Lessa, R., Lessa, B., Nery da Costa, A., Ruscitto do Prado, R., Oliveira-Campos, M. y Carvalho, D. (2014). Lifetime use of illicit drugs and associated factors among Brazilian schoolchildren, National Adolescent School-based Health Survey (PeNSE 2012). Revista Brasileira de Epidemiología, 17(1), 31-45. doi: http://dx.doi.org/10.1590/18094503201400050004

Losantos, M., Berckmans, I., Pieters, S., Dómic, J. y Loots, G. (2015). Resistiendo la exclusión: El significado del uso de inhalantes en diferentes contextos en jóvenes en situación de calle en la ciudad de La Paz. Ајауи, 13(2), 199-234. Recuperado de http://www.scielo.org.bo/pdf/rap/v13n2/v13 n2a05.pdf

Luo, T., Wang, J., Li, Y., Wang, X., Tan, L., Deng, Q., ... Hao, W. (enero, 2014). Stigmatization of people with drug dependence in China: A community-based study in Hunan province. Drug and Alcohol Dependence, 134(1), 285-289. doi: https://doi.org/10.1016/j.drugalcdep.2013.1 $\underline{0.015}$

Munro, M. (2015). The hijacked brain. Nature, 522, 46-47. doi: https://doi.org/10.1038/522S46a

Neto, C., Fraga, S. y Ramos, E. (2012). Consumo de substâncias ilícitas por adolescentes portugueses. Revista de Saúde Pública, 46(5), 1-7. Recuperado de http://www.scielo.br/pdf/rsp/v46n5/en 07.p df

Observatorio Social del Ecuador. (2016). Niñez y Adolescencia desde la intergeneracionalidad. Ecuador 2016. Recuperado de https://issuu.com/cnna ecuador/docs/ninez adolesc_intergeneracionalidad

Organización Mundial de la Salud. (2017). La dimensión de salud pública del problema mundial de las drogas. Proyecto de decisión presentado por la Argentina, Australia, Colombia, Costa Rica, Georgia, Montenegro, Noruega, los Países Bajos, Panamá, Sudáfrica, Suecia, Suiza, el Uruguay y Zambia. Recuperado de http://apps.who.int/gb/ebwha/pdf files/WH A70/A70_ACONF6-sp.pdf

Páramo, M. (julo, 2011). Factores de Riesgo y Factores de Protección en la Adolescencia: Análisis de Contenido a través de Grupos de Discusión. Terapia psicológica, 29(1), 8595. doi: https://dx.doi.org/10.4067/S071848082011000100009

Polgar, S. y Thomas, S. (2014). Introducción a la Investigación en Ciencias de la Salud (6 ${ }^{\mathrm{a}}$ ed.) Barcelona, España: Elsevier.

Potrikeeva, O., Suprun, N., Ispulova, S., Oleinik, E. y Slepukhina, G. (2017). Social Rehabilitation of Drug Addicts in Russia: Problems of Organization at the Current Stage and Prospects for Further Development [Resumen]. Man in India, 97(11), 489-500. Recuperado de https://elibrary.ru/item.asp?id=30493814

Rengel, D. (2005). La construcción social del 'otro'. Estigma, prejuicio e identidad en drogodependientes y enfermos de sida [Artículo 25]. Gazeta de Antropología, 21. Recuperado de http://www.ugr.es/ pwlac/G21_25Daniel $\underline{\text { Rengel_Morales.html }}$

Rojo-Mota, G. (2016). Terapia Ocupacional en adicciones: de la teoría a la 
práctica. Revista Chilena de Terapia Ocupacional, 16(1), 159-171. Recuperado de

https://revistaterapiaocupacional.uchile.cl/in dex.php/RTO/article/view/41953/44046

Secretaría Técnica de Prevención Integral de Drogas. (2017). Plan Nacional de Prevención Integral y Control del Fenómeno Socio Económico de las Drogas 2017-2021. Recuperado de https://es.scribd.com/document/385933369/ PLAN-NACIONAL-DE-PREVENCIONINTEGRAL-Y-CONTROL-DELFENOMENO-SOCIO-ECONOMICO-DELAS-DROGAS-2017-2021-281-29-pdf

Simões, C., Gaspar, M., Batista-Foguet, J. y Simons-Morton, B. (2014). Substance use across adolescence: do gender and age matter? Psicologia: Reflexão $e$ Crítica, 27(1), 179-188. doi: https://dx.doi.org/10.1590/S0102$\underline{79722014000100020}$

Touzé, G. (1995). La construcción social del problema droga. En P. Medina y G. Thivierge (Eds.), La investigación en toxicomanía: Estado y Perspectivas (pp.1339). Medellín, Colombia: UNLAM.

UNODC. (2017). Informe Mundial sobre las drogas 2017. Resumen, Conclusiones y Consecuencias en materia de Políticas. Recuperado de https://www.unodc.org/wdr2017/field/WD R_Booklet1_Exsum_Spanish.pdf

Vargas, Á. y Londoño, N. (2016). Creencias asociadas al consumo y dependencia de sustancias psicoactivas. Revista Katharsis, 21, 111-130. Recuperado de http://revistas.iue.edu.co/index.php/katharsi s/article/download/624/1053

Vargas, P., Parra, M., Arévalo, C., Cifuentes, L., Valero, J. y Sierra, M. (2015). Estructura y tipología familiar en pacientes con dependencia 0 abuso de sustancias psicoactivas en un centro de rehabilitación de adicciones en el Municipio de Chía, Cundinamarca. Revista Colombiana de Psiquiatría, 44(3), 166-176. Recuperado de http://www.redalyc.org/pdf/806/806430820 $\underline{07 . p d f}$

Tabla 1 Preguntas de entrevista semiestructurada

\begin{tabular}{|ll|}
\hline a) Datos sociodemográficos \\
1. & ¿Cuál es su edad? \\
2. & ¿Cuál es su dirección? \\
3. & ¿Se encuentra estudiando actualmente? \\
4. & ¿Hasta qué nivel educativo llegó? \\
5. & ¿Cuál es la ocupación de su madre? \\
6. & ¿Cuáles son ocupación de su padre? \\
& de su familia?
\end{tabular}

b) Historia pasada del consumo de droga

7. ¿Qué edad tenía usted cuando consumió por primera vez droga?

8. ¿Dónde fue el primer consumo de droga?

9. ¿Quién le ofreció por primera vez la droga?

c) Motivación para el consumo de droga

10. ¿Por qué accediste al consumo de drogas?

d) Vida de la persona que consume droga

11. ¿Cómo es tu vida familiar desde que consumes droga?

12. ¿Cómo es tu vida social desde que consumes droga?

13. ¿Cómo es tu vida personal desde que consumes droga?

e) Ventajas y desventajas percibidas por el consumo de drogas

14. ¿Qué beneficios crees que te da el consumo de drogas?

15. ¿Qué perjuicios crees que te da el consumo de droga?

f) Perspectivas de la vida a futuro

16. ¿Qué futuro piensas que le espera a tu vida por el consumo de droga?

17. ¿Cuál crees que sería la solución para poder salir del consumo de drogas?

Fuente: Elaboración propia. 

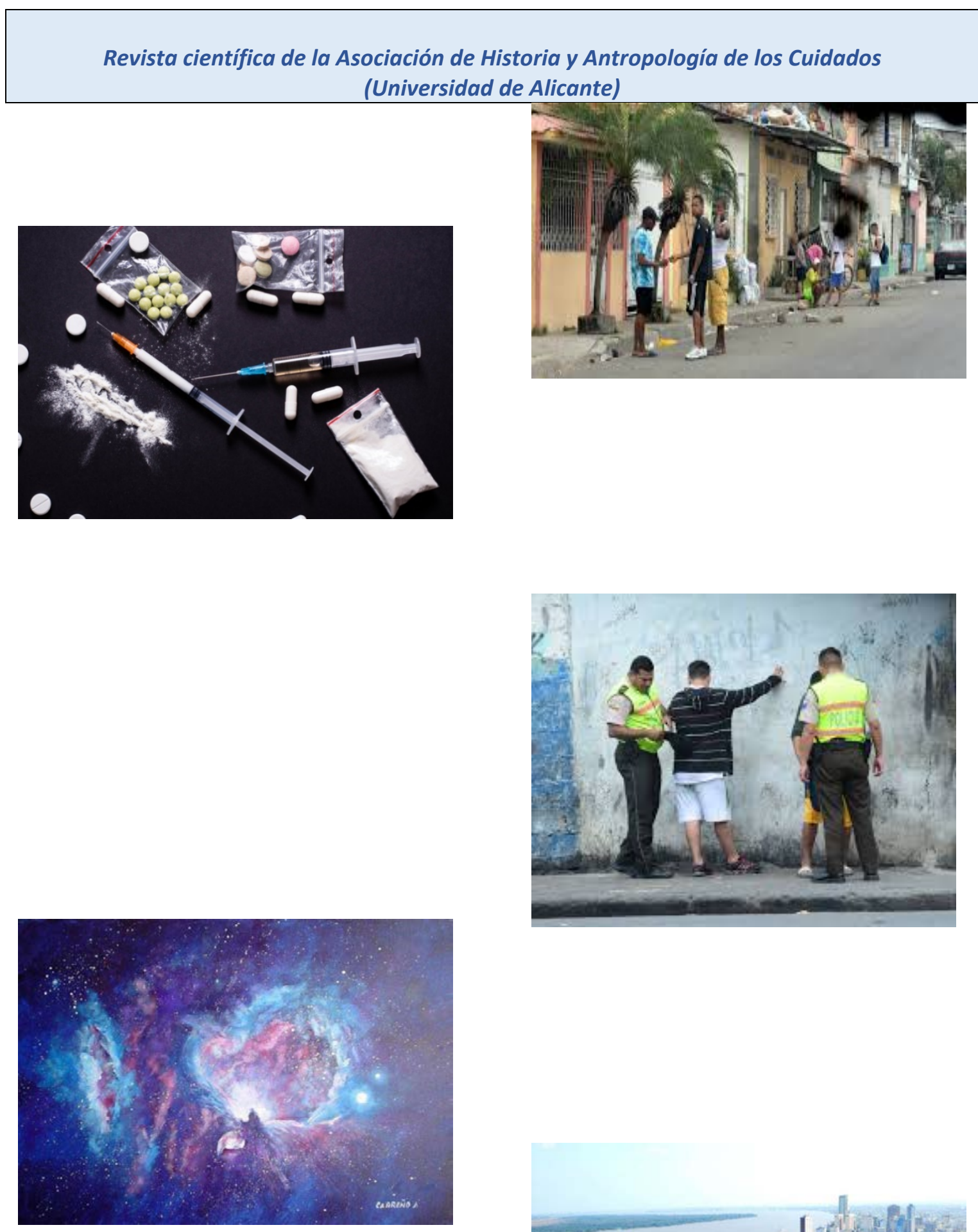

Constelacion de Orion. Pintura de Antonio Carreño

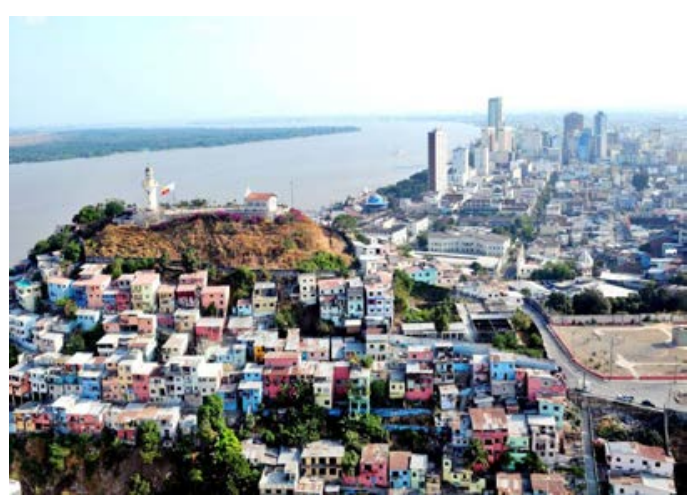

